'Departamento de Salud Pública, Escuela de Medicina, Pontificia Universidad Católica de Chile. ${ }^{2}$ Escuela de Medicina, Pontificia Universidad Católica de Chile. ${ }^{3}$ Departamento de Gastroenterología, Pontificia Universidad Católica de Chile. ${ }^{4}$ Escuela de Construcción Civil, Facultad de Ingeniería, Pontificia Universidad Católica de Chile ${ }^{5}$ Centro de Educación Médica Escuela de Medicina, Pontificia Universidad Católica de Chile. aPsicólogo/a. bBioquímico/a. "Sociólogo/a. dEstadístico/a.

Proyecto de investigación financiado por la Dirección de Postgrado de la Pontificia Universidad Católica de Chile y por el Fondo Nacional de Desarrollo Científico y Tecnológico (FONDECYT) proyectos № 1120652

(A.R.) y No 1120534 (M.B.)

Recibido el 23 de abril de 2013 aceptado el 25 de junio de 2013

Correspondencia a:

Dr. Arnoldo Riquelme, MMedEd

Departamento de

Gastroenterología.

Centro de Educación Médica.

Escuela de Medicina. Pontificia Universidad Católica de Chile.

Marcoleta 367, Santiago, Chile.

Casilla 114-D. Santiago, Chile.

Teléfonos: 56-2-23543845 y

56-9-81361686

E-mail: a.riquelme.perez@gmail.

com

\section{Identificación de las necesidades de capacitación docente de los jefes de programa de especialización médica}

\author{
CRISTIAN A. HERRERA ${ }^{1}$, IAN NIKLITSCHEK ${ }^{2}$, MARGARITA PIZARRO ${ }^{3, b}$, \\ NANCY SOLÍS 3 ,b, TRINIDAD OLIVOS ${ }^{3, a}$, VIVIANA ROJAS 3 ,a, \\ LORENA ETCHEBERRY ${ }^{3, \mathrm{c}}$, HORACIO RIVERA ${ }^{4, a}$, ESTRELLA MUÑOZ ${ }^{3, a}$, \\ MARCELA BITRAN ${ }^{5}$, OSLANDO PADILLA ${ }^{1, \mathrm{~d}}$, ARNOLDO RIQUELME ${ }^{3,5}$

\section{Identifying the main training needs of postgraduate medical program managers}

Background: Training of postgraduate medical specialty program managers (PMSPM) is essential for the proper development of their programs. Aim: To identify the main training needs of PMSPM at a medical school. Material and Methods: A mixed-methodology approach was implemented including focus group/interviews and the administration of the Program Managers Training Needs Assessment Questionnaire (PROMANAQ) developed by an expert panel with 59 items (with two sections: relevance/performance-selfperception). Higher priority was assigned to items with high relevance and low performance. Results: Forty five PMSPM completed the PROMANAQ (81.8\% response rate). Both sections of PROMANAQ were highly reliable (Cronbach alpha of 0.95/0.97 for relevance/performance-self-perception, respectively). The items with higher priority value were evaluation of clinical educators, evaluation of teaching programs and accreditation of programs. Ten PMSPM were included in the focus group (18.2\% of the universe). The findings of the qualitative component were concordant with the areas explored in the questionnaire. Conclusions: The PROMANAQ is valid and reliable to identify the training needs of PMSPM. The views of PMSPM must be taken into account for faculty development planning.

(Rev Med Chile 2013; 141: 1126-1135).

Key words: Education, medical, graduate; Educational measurement; Questionnaires.
L os programas de especialidades médicas han tenido un gran desarrollo en los últimos años, con el advenimiento de nuevas tecnologías y la necesidad de mayor conocimiento y entrenamiento en el manejo específico de enfermedades complejas ${ }^{1}$. El desarrollo de estos programas se basa en la capacidad de transmisión de conocimientos, destrezas y actitudes de parte de especialistas altamente calificados en el manejo de pacientes, pero que por lo general, no tienen una formación profesional en los aspectos relacionados con la docencia ${ }^{2}$. La capacitación docente se refiere a un amplio espectro de actividades que las instituciones usan para apoyar a los facultativos en el adecuado desarrollo de roles relacionados con la enseñanza, investigación y administración ${ }^{3-5}$.

En las especialidades médicas, la mayoría de los programas de formación enfocan la capacitación docente en el mejoramiento de la enseñanza de postgrado basado en pacientes, control de sesiones de pequeño grupo (seminarios) y reuniones clíni- 
cas, entrega de retroalimentación (feedback) y evaluación ${ }^{6}$. Sin embargo, otras áreas del quehacer docente que se relacionan con inter-profesionalismo, habilidades comunicacionales, diseño curricular o la conducción de procesos de acreditación de programas, son habitualmente ignoradas o poco desarrolladas ${ }^{7}$. En ese sentido, la capacitación docente debe incluir la transmisión de metodología apropiada para educar a los docentes en nuevos conceptos en educación médica, los que incluyen: el desarrollo curricular de programas de acuerdo a competencias ${ }^{8,9}$; el uso de metodologías de enseñanza y aprendizaje independiente (portafolio $)^{10}$; simulación de procedimientos prácticos ${ }^{11}$; enseñanza a distancia y en pequeños grupos; mentoría y retroalimentación efectiva; evaluación de programas docentes ${ }^{12}$; la relevancia del ambiente educacional ${ }^{13}$; y evaluación de conocimientos (pruebas escritas), destrezas clínicas (exámenes objetivos estructurados $)^{14} \mathrm{y}$ destrezas en el sitio de trabajo (pautas de observación directa) ${ }^{15}$.

La población objetiva a la cual se orienta el programa es de particular interés ya que existen distintos perfiles o roles que requieren capacitación focalizada. Algunos docentes están encargados del diseño y desarrollo curricular, implementación de guías de estudio, desarrollo de instrumentos de evaluación (como es el caso de los jefes de programas) que comparten necesidades comunes y que, a su vez, son distintas a las de los profesores clínicos, que en general requieren más capacitación en metodologías de docencia y formas de entrega de retroalimentación a los residentes en entrenamiento clínico y de procedimientos médico-quirúrgicos ${ }^{16}$. Este proceso debe considerar las redes profesionales ${ }^{17}$, aspectos culturales, disponibilidad horaria de los docentes, tiempos protegidos, clima organizacional, así como los reconocimientos y estímulos adecuados para que el programa de capacitación sea exitoso ${ }^{6}$.

En este contexto, para poder diseñar un plan de capacitación de jefes de programas de especialidades médicas (JPEM) se hace necesario un proceso de identificación de las necesidades que ellos tienen, de manera de ofrecer un programa variado que responda a sus expectativas en términos de su relevancia, aplicabilidad y factibilidad. El objetivo de este estudio fue identificar las principales necesidades de capacitación de los JPEM en la Facultad de Medicina de la Pontificia Universidad Católica de Chile (FM-PUC), con el fin de implementar -a futuro- un plan de capacitación docente focalizado en estas necesidades y preferencias.

\section{Métodos}

El estudio consideró una metodología mixta, es decir, complementó una parte cuantitativa y otra cualitativa, usando la técnica del protocolo de triangulación ${ }^{18}$ para la interpretación de los resultados. La sección cuantitativa se destinó al diseño y construcción del Cuestionario de Análisis de Necesidades de Capacitación para Jefes de Programa (CAJEP), el cual fue realizado por un equipo de expertos multidisciplinario en base a una búsqueda en la literatura relacionada al tema, tomando como marco conceptual los criterios de excelencia del educador médico de Hesketh et al. ${ }^{19}$. El instrumento resultante fue aplicado a los JPEM de la FM-PUC, consultándoles tanto por el grado importancia que le asignaban como por la autopercepción de su actual desempeño en cada ítem. Aquellos ítems que fueron considerados de alta importancia y pobre desempeño, obtuvieron la mayor calificación en la escala de Likert de 5 puntos. Se calculó un puntaje de priorización, en base al producto de ambos puntajes para cada ítem, con el cual se generó un ranking de priorización ${ }^{20}$. Se realizó análisis de escalamiento multidimensional para luego medir la consistencia interna de cada sección (confiabilidad) mediante el método de Cronbach alfa ${ }^{21}$.

Para el componente cualitativo, se realizó una entrevista grupal focal con jefes de programa y una entrevista semi-estructurada a una alta autoridad de la Dirección de Postgrado de la FM-PUC. Los datos fueron analizados utilizando la metodología de la Teoría Fundada ${ }^{22}$, mediante una codificación abierta para identificar las categorías de conceptos que emergieron de la entrevista grupal focal. Para garantizar la eficiencia y sistematicidad del análisis se utilizó el software Atlas.Ti ${ }^{\circledR}$. Los resultados fueron sometidos a los criterios de control de calidad propuestos por Ruiz ${ }^{23}$. El proyecto fue aprobado por el comité de ética de la FM-PUC y todos los participantes firmaron consentimiento informado al participar en las entrevistas individual/grupal focal. Los resultados de las entrevistas individual/grupal focal fueron confidenciales y las encuestas fueron respondidas de manera anónima. 


\section{Resultados}

\section{Análisis cuantitativo}

Cuarenta y cinco jefes de programa completaron la versión final del CAJEP que contenía 59 ítems (tasa de respuesta de $81,8 \%$ ), de los cuales $73 \%$ fueron hombres, $44,4 \%$ tenían entre 41 y 50 años, y 51,1\% tenían un diplomado en educación médica (Tabla 1).

Los ítems con mayor puntaje de priorización fueron la evaluación de docentes clínicos, la evaluación de programas docentes y la acreditación de programas (Tabla 2).

El análisis de confiabilidad de las secciones del cuestionario mostró que la sección que consultó acerca de la relevancia de cada ítem, obtuvo un coeficiente de Cronbach alfa de 0,95 y el que preguntó por auto-percepción de desempeño fue de 0,97.
Tabla 1. Descripción de los jefes de programa encuestados con el CAJEP

\begin{tabular}{lr|} 
Característica & Respuestas (n = 45) \\
Género & \\
Masculino & $33(73,3 \%)$ \\
Femenino & $12(26,7 \%)$ \\
Edad & \\
$31-40$ & $8(17,8 \%)$ \\
$41-50$ & $20(44,4 \%)$ \\
$51-60$ & $13(28,9 \%)$ \\
$>60$ & $4(8,9 \%)$ \\
Desempeño docente & \\
Postgrado medicina & $39(86,7 \%)$ \\
Pregrado medicina & $26(57,8 \%)$ \\
Multiprofesional & $15(33,3 \%)$ \\
Otro & $12(26,7 \%)$ \\
\hline
\end{tabular}

CAJEP: Cuestionario de Análisis de Necesidades de Capacitación para Jefes de Programa.

Tabla 2. Áreas de capacitación del CAJEP ordenado por ranking de priordad de acuerdo a la evaluación de importancia y desempeño de los Jefes de Programa de la FM-PUC

\begin{tabular}{|c|c|c|c|}
\hline $\begin{array}{l}\text { Ranking de } \\
\text { prioridad }\end{array}$ & Área de capacitación y orden de ítems & $\begin{array}{l}\text { Importancia } \\
\text { atribuida }\end{array}$ & $\begin{array}{l}\text { Desempeño } \\
\text { autopercibido }\end{array}$ \\
\hline 1 & 32. Evaluación de docentes clínicos & 4,28 & 3,02 \\
\hline 2 & 12. Evaluación de programas docentes & 4,38 & 3,07 \\
\hline 3 & 26. Acreditación de programas & 4,13 & 2,93 \\
\hline 4 & 16. Investigación en docencia & 3,96 & 2,69 \\
\hline 5 & 30. Portafolio & 3,65 & 2,61 \\
\hline 6 & 27. Currículo basado en competencias & 4,27 & 3,14 \\
\hline 7 & 21. Qué hacer con alumnos en problemas & 4,29 & 3,24 \\
\hline 8 & 13. Bases de la teoría educacional & 3,31 & 2,39 \\
\hline 9 & 4. Docencia en destrezas clínicas/paciente simulado & 4,09 & 3,08 \\
\hline 10 & 10. Diseño de cursos/diseño curricular & 4,07 & 3,05 \\
\hline 11 & 25. Enseñanza y aprendizaje de procedimientos en ambientes simulados & 3,57 & 2,67 \\
\hline 12 & 20. Manejo de crisis & 4,29 & 3,31 \\
\hline 13 & 31. Capacitación docente & 4,09 & 3,22 \\
\hline 14 & 14. Aspectos éticos/legales en docencia & 3,73 & 2,93 \\
\hline 15 & 45. Diseño de guías de estudio & 3,93 & 3,11 \\
\hline 16 & 22. Programas basados en competencias & 4,27 & 3,36 \\
\hline 17 & $\begin{array}{l}\text { 58. Evaluación de destrezas clínicas/Examen Objetivo Clínico Estructura- } \\
\text { do (ECOE) }\end{array}$ & 3,73 & 2,98 \\
\hline 18 & 19. Liderazgo & 4,20 & 3,40 \\
\hline 19 & 33. Desarrollo profesional continuo relacionado con docencia & 4,18 & 3,38 \\
\hline 20 & 49. Aprendizaje a distancia & 3,18 & 2,58 \\
\hline
\end{tabular}




\begin{tabular}{|c|c|c|c|}
\hline 21 & 9. Dar y recibir retroalimentación (feedback) & 4,51 & 3,62 \\
\hline 22 & 44. Diseño de textos, manuales y protocolos & 3,80 & 3,16 \\
\hline 23 & 15. Educación médica basada en evidencia & 4,09 & 3,33 \\
\hline 24 & 57. Manejo y administración en salud & 3,57 & 2,95 \\
\hline 25 & 42. Autoevaluación de destrezas & 3,78 & 3,24 \\
\hline 26 & 47. Proceso de admisión y selección de postulantes & 4,11 & 3,47 \\
\hline 27 & 5. Mentoría/apoyo alumnos & 4,18 & 3,48 \\
\hline 28 & 11. Evaluación de conocimientos (pruebas escritas) & 4,29 & 3,60 \\
\hline 29 & 17. Educación multiprofesional/interprofesional & 3,47 & 2,91 \\
\hline 30 & 37. Consejería a estudiantes & 3,89 & 3,36 \\
\hline 31 & 35. Manejo de comportamientos desafiantes (conductas peligrosas) & 3,69 & 3,16 \\
\hline 32 & 43. Manejo del tiempo & 3,84 & 3,31 \\
\hline 33 & 41. Estilos de enseñanza y aprendizaje & 3,47 & 3,02 \\
\hline 34 & 51. Enseñanza y aprendizaje de actitudes profesionales & 4,13 & 3,56 \\
\hline 35 & 18. Educación y aspectos interculturales & 3,11 & 2,70 \\
\hline 36 & 28. Profesionalismo & 4,38 & 3,73 \\
\hline 37 & 24. Enseñanza en sala espejo & 2,91 & 2,34 \\
\hline 38 & 36. Mantenerse actualizado en educación médica & 3,93 & 3,38 \\
\hline 39 & 6. Redacción de objetivos & 4,02 & 3,60 \\
\hline 40 & 23. Evaluación en el sitio de trabajo & 4,02 & 3,45 \\
\hline 41 & 29. Ambiente educacional & 4,09 & 3,70 \\
\hline 42 & 50. Reglamento y medidas disciplinarias & 3,18 & 3,02 \\
\hline 43 & 39. Creación de material audiovisual & 3,96 & 3,64 \\
\hline 44 & 2. Enseñanza y aprendizaje en grupos pequeños & 4,47 & 3,93 \\
\hline 45 & 7. Supervisión docente & 4,33 & 3,91 \\
\hline 46 & 34. Aprendizaje a lo largo de la vida & 4,09 & 3,73 \\
\hline 47 & 52. Responsabilidad social & 3,80 & 3,59 \\
\hline 48 & $\begin{array}{l}\text { 54. Requerimientos del Ministerio de Salud y Garantías Explícitas en } \\
\text { Salud }\end{array}$ & 3,58 & 3,42 \\
\hline 49 & 55. Regulaciones de la Facultad y de la Universidad & 3,38 & 3,38 \\
\hline 50 & 3. Docencia en ambientes hospitalarios & 4,18 & 3,75 \\
\hline 51 & 59. Derechos, responsabilidades e información al paciente & 3,73 & 3,67 \\
\hline 52 & 48. Enseñanza y aprendizaje en el sitio de trabajo & 3,80 & 3,66 \\
\hline 53 & 40. Enseñanza en la comunidad & 2,96 & 3,02 \\
\hline 54 & 38. Comunicación oral & 4,07 & 3,91 \\
\hline 55 & 8. Docencia en seminarios & 4,11 & 3,96 \\
\hline 56 & 53. Trabajo en equipo de salud & 4,09 & 3,98 \\
\hline 57 & 56. Aceptar evaluaciones y críticas constructivas & 3,89 & 3,86 \\
\hline 58 & 46. Acceso a información & 4,00 & 3,98 \\
\hline 59 & 1. Docencia a grupos grandes y como dictar conferencias & 3,23 & 3,82 \\
\hline
\end{tabular}

CAJEP: Cuestionario de Análisis de Necesidades de Capacitación para Jefes de Programa. FM-PUC: Facultad de Medicina de la Pontificia Universidad Católica de Chile. 
Sobre la metodología de enseñanza preferida, $51 \%$ dice optar por aprendizaje mixto (presencial y a distancia), 44\% sólo talleres, y 13\% sólo educación a distancia. Las preferencias se pueden ver en la Figura 1.

En cuanto a los horarios de preferencia por parte de los JPEM, podemos evidenciar que la gran mayoría prefiere tener las actividades de capacitación durante el horario laboral de lunes a viernes, aunque $20 \%$ preferiría que fuese un sábado por la mañana (Figura 2).

\section{Análisis cualitativo}

El grupo focal incluyó al 18,2\% del universo de JPEM (10 de 55 programas de especialidades o subespecialidades médicas). De lo obtenido de este grupo focal más la entrevista en profundidad sostenida con un alto directivo de la Dirección de Postgrado, se optó por clasificar los temas relevantes en cinco áreas o categorías y en cada una se identificaron sub categorías (Tabla 3). El área de comunicación se refiere a diferentes aspectos del fenómeno comunicacional que atraviesa tanto los aspectos formativos como las relaciones entre las personas en términos amplios. El ámbito de métodos de evaluación del aprendizaje aborda la necesidad de conocer con mayor precisión diferentes formas de constatar que el aprendizaje se ha instalado. La gestión de la formación trata sobre los aspectos de manejo tanto administrativo como de dirección, motivación y compromiso con los médicos en formación de especialidad, junto a las restricciones y apoyos institucionales que enfrentan los JPEM. El profesionalismo plantea
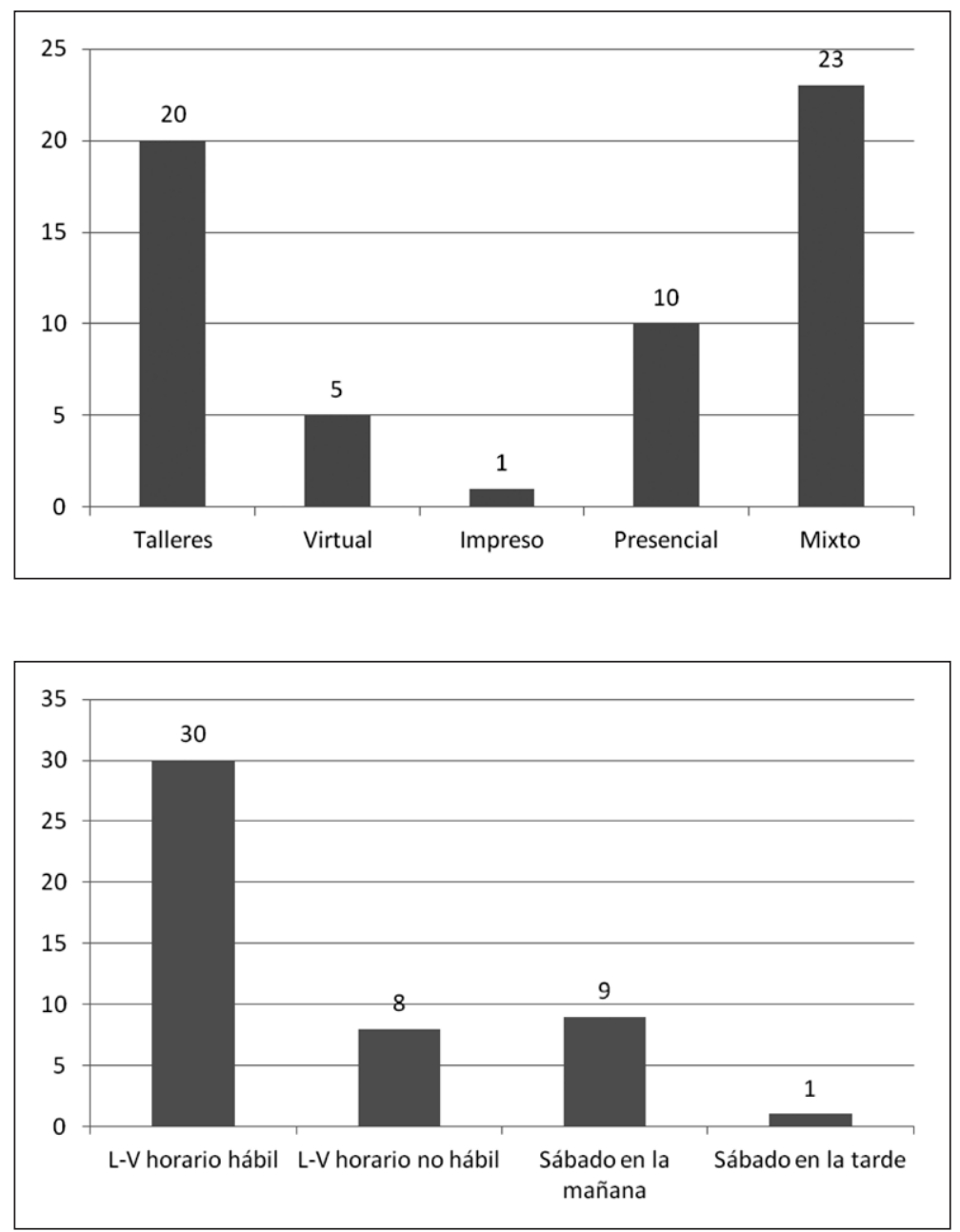

Figura 1. Preferencias de los jefes de programa en cuanto a metodología de enseñanza $(n=45)$.
Figura 2. Preferencias de los jefes de programa en cuanto al horario para realizar las capacitaciones $(n=45)$. L-V: Lunes a viernes. 
Tabla 3. Categorías y sub-categorías identificadas en el componente cualitativo sobre las necesidades de capacitación de los jefes de programa

\begin{tabular}{|c|c|c|}
\hline \multirow{2}{*}{$\begin{array}{l}\text { Categorías } \\
\text { 1. Comunicación }\end{array}$} & \multicolumn{2}{|c|}{ Sub-categorías } \\
\hline & 1.2 & Con pares, con pacientes, con superiores, con otros profesionales de la salud \\
\hline & 1.2 & Empatía \\
\hline & 1.3 & Flexibilidad \\
\hline & 1.4 & Asertividad \\
\hline & 1.5 & Trato interpersonal, respeto por pares y residentes/Modelaje \\
\hline & 1.6 & Generar confianza, capacidad de escucha, no descalificar al residente \\
\hline \multirow{4}{*}{$\begin{array}{l}\text { 2. Métodos de evaluación } \\
\text { del aprendizaje }\end{array}$} & 2.1 & Retroalimentación estructurada y bidireccional \\
\hline & 2.2 & Portafolio \\
\hline & 2.3 & Evaluación en sitio de trabajo/Construcción pautas \\
\hline & 2.4 & Diferenciar evaluación en becas grandes vs pequeñas \\
\hline \multirow[t]{11}{*}{ 3. Gestión de la formación } & 3.1 & Enfrentamiento de situaciones complejas \\
\hline & 3.2 & Habilidades de coordinación, de articulación \\
\hline & 3.3 & Liderazgo situacional, con residentes, con pares \\
\hline & 3.4 & $\begin{array}{l}\text { Entrevista de selección: desarrollo de criterios y pautas, reconocimiento de factores que } \\
\text { puedan dificultar o facilitar al postulante un buen desarrollo de su especialización }\end{array}$ \\
\hline & 3.5 & Clima de aprendizaje y Clima laboral \\
\hline & 3.6 & $\begin{array}{l}\text { Modificación de la cultura organizacional que considera "natural" la sobrecarga de } \\
\text { trabajo del médico }\end{array}$ \\
\hline & 3.7 & $\begin{array}{l}\text { Capacidad para percibir las diferencias individuales, apreciarlas, no discriminar en función } \\
\text { de ellas y de ajustarse a estándares técnicos de la formación considerando esa diversidad }\end{array}$ \\
\hline & 3.8 & Afrontamiento constructivo del conflicto \\
\hline & 3.9 & Formalización oportuna de los cambios que se introducen en los programas \\
\hline & 3.10 & Profesionalización del rol de jefe de programa, dedicación exclusiva \\
\hline & 3.11 & $\begin{array}{l}\text { Evidenciar los apoyos institucionales y otros con que cuentan los jefes de programa para } \\
\text { enfrentar sus funciones (por ejemplo, asesoría del psiquiatra) }\end{array}$ \\
\hline \multirow[t]{2}{*}{ 4. Profesionalismo } & 4.1 & Responsabilidad y ética cotidianas \\
\hline & 4.2 & $\begin{array}{l}\text { Responsabilidad del médico especialista ante la sociedad chilena (más allá de la FM-PUC } \\
\text { y la práctica clínica privada) }\end{array}$ \\
\hline 5. Idiomas & 5.1 & Idioma inglés \\
\hline
\end{tabular}

FM-PUC (Facultad de Medicina de la Pontificia Universidad Católica de Chile).

una reflexión acerca de las implicancias éticas de las actuaciones y decisiones diarias del especialista, y a crear conciencia de su compromiso con el desarrollo y la salud a nivel país. Y por último, el idioma se refiere a la necesidad de comprender y expresarse en inglés.

Para aplicar la técnica del protocolo de triangulación, elaboramos una 'matriz de codificación de convergencias', donde se pueden identificar de mejor manera lo que aporta cada metodología a la exploración de las necesidades de capacitación de los JPEM (Tabla 4). Los acuerdos se refieren a los puntos que fueron identificados concordantemente en ambas metodologías; los acuerdos parciales a los hallazgos que abordan una misma área pero tienen alguna disimilitud; las disonancias a los puntos en los cuales los resultados fueron divergentes o contradictorios; y los silencios a los hallazgos que una metodología encontró u obtuvo y la otra no. 
Tabla 4. Matriz de codificación de convergencias entre las metodologías cuantitativa y cualitativa

\begin{tabular}{|c|c|c|}
\hline & Cuantitativo (CAJEP) ${ }^{a}$ & $\begin{array}{l}\text { Cualitativo (entrevista individual y grupo } \\
\text { focal)b }^{b}\end{array}$ \\
\hline \multirow[t]{7}{*}{ Acuerdos } & $\begin{array}{l}\text { 9. Dar y recibir retroalimentación (feedback) } \\
\text { 30. Portafolio } \\
\text { 33. Evaluación en sitio de trabajo } \\
\text { 48. Enseñanza y aprendizaje en sitio de trabajo }\end{array}$ & $\begin{array}{l}\text { 2.1 Retroalimentación estructurada y bidireccional } \\
\text { 2.2 Portafolio } \\
\text { 2.3 Evaluación en sitio de trabajo/construcción pautas }\end{array}$ \\
\hline & 19. Liderazgo & $\begin{array}{l}\text { 3.2 Habilidades de coordinación, de articulación } \\
\text { 3.3 Liderazgo situacional, con residentes, con pares }\end{array}$ \\
\hline & 47. Proceso de admisión y selección de postulantes & $\begin{array}{l}\text { 3.4 Entrevista de selección: desarrollo de criterios } \\
\text { y pautas }\end{array}$ \\
\hline & $\begin{array}{l}\text { 20. Manejo de crisis } \\
\text { 21. Qué hacer con alumnos en problemas }\end{array}$ & $\begin{array}{l}\text { 3.1 Enfrentamiento de situaciones complejas } \\
\text { 3.8 Afrontamiento constructivo del conflicto }\end{array}$ \\
\hline & 29. Ambiente educacional & 3.5 Clima de aprendizaje y Clima laboral \\
\hline & $\begin{array}{l}\text { 28. Profesionalismo } \\
\text { 51. Enseñanza y aprendizaje de actitudes } \\
\text { profesionales }\end{array}$ & 4.1 Responsabilidad y ética cotidianas \\
\hline & 52. Responsabilidad social & $\begin{array}{l}\text { 4.2 Responsabilidad del médico especialista ante la } \\
\text { sociedad chilena (más allá de la FM-PUC y la } \\
\text { práctica clínica privada) }\end{array}$ \\
\hline \multirow[t]{3}{*}{$\begin{array}{l}\text { Acuerdos } \\
\text { parciales }\end{array}$} & $\begin{array}{l}\text { 38. Comunicación oral } \\
\text { 5. Mentoría/apoyo alumnos } \\
\text { 56. Aceptar evaluaciones y críticas constructivas } \\
\text { 37. Consejería a estudiantes } \\
\text { 53. Trabajo en equipo de salud }\end{array}$ & $\begin{array}{l}\text { 1. Comunicación en sentido amplio: con residentes, } \\
\text { con pares, con pacientes, con superiores, con } \\
\text { otros profesionales de la salud, incluye empatía, } \\
\text { asertividad, flexibilidad, }\end{array}$ \\
\hline & $\begin{array}{l}\text { Los temas referidos a Docencia, Evaluación del } \\
\text { aprendizaje aparecen con detalle y corresponden } \\
\text { a los ítems: } 2,3,4,6,7,8,10,11,12,13,14,15 \text {, } \\
\begin{array}{l}16,17,18,22,24,25,26,27,31,32,36,39,40 \\
41,42,44,45,49,58\end{array}\end{array}$ & $\begin{array}{l}\text { 2. Métodos de evaluación del aprendizaje, } \\
\text { aparecen con menos detalle en la investigación } \\
\text { cualitativa y están registrados en Acuerdos }\end{array}$ \\
\hline & $\begin{array}{l}\text { 33. Desarrollo profesional continuo relacionado } \\
\text { con docencia }\end{array}$ & 3.10 Profesionalizar rol JPEM \\
\hline Disonancias & $\begin{array}{l}\text { 1. Docencia a grupos grandes y cómo dictar } \\
\text { conferencias }\end{array}$ & \\
\hline Silencios & $\begin{array}{l}\text { 34. Aprendizaje a lo largo de la vida } \\
\text { 35. Manejo de comportamientos desafiantes } \\
\text { (conductas peligrosas) } \\
\text { 43. Manejo del tiempo } \\
\text { 46. Acceso a información } \\
\text { 50. Reglamento y medidas disciplinarias } \\
\text { 54. Requerimientos del Ministerio de Salud y } \\
\text { Garantías Explícitas en Salud } \\
\text { 55. Regulaciones de la Facultad y de la Universidad } \\
\text { 57. Manejo y administración en salud } \\
\text { 59. Derechos, responsabilidades e información al } \\
\text { paciente }\end{array}$ & $\begin{array}{l}\text { 2.4 Diferenciar evaluación en becas grandes vs } \\
\text { pequeñas } \\
\text { 3.6 Modificación de la cultura organizacional que } \\
\text { considera "natural" la sobrecarga de trabajo del } \\
\text { médico } \\
\text { 3.7 Capacidad para percibir las diferencias indivi- } \\
\text { duales, apreciarlas, no discriminar en función } \\
\text { de ellas y de ajustarse a estándares técnicos de } \\
\text { la formación considerando esa diversidad } \\
\text { 3.9 Formalización de cambios a programas } \\
\text { 3.11 Visibilizar apoyos institucionales y otros a JPEM } \\
\text { 5.1 Idioma inglés }\end{array}$ \\
\hline
\end{tabular}

aítem del CAJEP. 'Subcategorías de la Tabla 3. CAJEP: Cuestionario de Análisis de Necesidades de Capacitación para Jefes de Programa. JPEM: Jefes de programas de especialidades médicas. FM-PUC: Facultad de Medicina de la Pontificia Universidad Católica de Chile. 


\section{Discusión}

La capacitación docente es un área de la educación médica de creciente interés debido al impacto educacional que puede generar en una institución académica. Sin embargo, son pocos los estudios que incluyen un análisis de necesidades y un diseño programático acorde a la información recolectada en estudios basados en metodología cualitativa o cuantitativa ${ }^{20}$. El presente estudio ofrece una interesante aproximación, ya que considera la opinión de los JPEM de la FMPUC, mediante el empleo de una metodología mixta. El estudio comenzó con la creación de un instrumento con validez de contenido y alta confiabilidad, que puede ser utilizado en instituciones de habla hispana para realizar un análisis de necesidades de sus JPEM o profesores clínicos de programas de especialidades médicas, ya que el CAJEP incluye un amplio espectro de competencias del educador médico de excelencia ${ }^{19}$. Los aspectos susceptibles de capacitación docente que obtuvieron mayor puntaje de priorización fueron la evaluación de docentes clínicos, la evaluación de programas docentes y la acreditación de programas. Sin embargo, esta medición constituye un diagnóstico del momento en el cual se aplica la CAJEP, y nuestra intención es aplicarla anualmente para obtener una información dinámica en el tiempo. Sobre la metodología de enseñanza preferida, la mayoría de los JPEM prefiere un modelo de aprendizaje mixto (presencial y a distancia), durante el horario laboral. Con la información obtenida en este estudio transversal, ya conocemos los aspectos a considerar dentro de los contenidos de la capacitación, la metodología de aprendizaje y el horario preferido por los encuestados. En cuanto a su implementación, los formatos educacionales más frecuentemente utilizados incluyen talleres y seminarios, cursos cortos y sabáticos. Los talleres son las actividades más populares por su flexibilidad y capacidad de promover aprendizaje activo ${ }^{24,25}$. Las jornadas de capacitación deben promover actividades descentralizadas ${ }^{26}$, mentoría ${ }^{4}$ o acompañamiento por pares $^{27}$, aprendizaje auto-dirigido y a distancia ${ }^{1}$. Si bien, las jornadas de capacitación deben considerar temas prioritarios para todos los JPEM, se deben incluir oportunidades de capacitación para JPEM con necesidades particulares, como por ejemplo, el acompañamiento de jefes de programas que serán sometidos al proceso de acreditación en el futuro cercano ${ }^{24}$.

Para el adecuado diseño e implementación de un programa de capacitación docente, se debe partir por la comprensión y entendimiento de la cultura organizacional de la institución y se debe estar atento a las barreras institucionales que dificultan la asistencia de los docentes a actividades de capacitación ${ }^{25,28}$. El contexto cultural se puede utilizar para promover los esfuerzos de capacitación docente. Por ejemplo, la capacitación docente en el tiempo de una reforma curricular o educacional puede adquirir mayor relevancia ${ }^{29}$. También es importante evaluar si existe el apoyo institucional para llevar a cabo las actividades de capacitación docente, asegurándose que exista disponibilidad de recursos y tiempos protegidos, tanto para quienes organicen las actividades, como para los académicos que asistan ${ }^{30}$. En este sentido, el equipo de capacitación obtuvo el apoyo de la dirección de postgrado y del director de escuela, facilitando el desarrollo del proyecto, espacios físicos y acceso a los JPEM.

El presente trabajo incluyó la triangulación de información cualitativa y cuantitativa de parte de los JPEM de la FM-PUC. Esta información coincide con la percepción del ambiente educacional de residentes y los aspectos que coinciden en la triangulación (acuerdos) son: programas y guías de estudio, feedback, consejería, mentoría, permisos y financiamiento para asistir a congresos, regulación de las horas de trabajo, tiempo protegido para instancias académicas, entrenamiento y evaluación de destrezas clínicas ${ }^{31,32}$. Debemos destacar que la triangulación de información es de suma relevancia porque existe coincidencia en la mayoría de los aspectos evaluados, pero también se observan disonancias y silencios de aspectos emergentes en la investigación cualitativa, como por ejemplo, la importancia del manejo del idioma inglés, como competencia requerida entre los JPEM, que pudiera llegar a ser parte de la descripción del cargo. La evaluación de un programa de capacitación docente es uno de los aspectos más importantes en el proceso de desarrollo e implementación del programa y se debe comprender que dicha evaluación es más que un mero ejercicio académico, ya que los resultados que emanen de éstos, servirán para futuros cambios en el diseño, con especial impacto en los formatos de entrega que promuevan un proceso de enseñanza y aprendizaje más eficaz ${ }^{33}$. 
Steinert estableció, hace más de 10 años, que la capacitación docente debe ser la guía que promueva los procesos educativos de una institución y debe cumplir con un rol de modelaje de todo lo que se hace al interior de dicha institución ${ }^{34}$. La evaluación de los niveles de Kirkpatrick ${ }^{35}$ es de gran utilidad para conceptualizar y estructurar la evaluación de logros que incluyen 4 niveles desde la reacción y aprendizaje, hasta cambios en la conducta de los participantes y resultados, que implican cambios en el sistema organizacional, con impacto en el cuidado del paciente o en el que aprende. Desde el año 2008, el Centro de Educación Médica de la FM-PUC, implementó un programa longitudinal de capacitación para JPEM. Dicho programa incluyó 6 cursos que contemplaron los aspectos genéricos más importantes de la educación médica, que asegura un conocimiento basal de las áreas referentes al desarrollo curricular, metodologías de enseñanza y aprendizaje, así como conceptos relacionados con evaluación ${ }^{36}$. Treinta jefes de programa, que corresponde a cerca de $50 \%$, asistieron a este curso abreviado de capacitación y al final del curso de capacitación, se demostró impacto educacional en los 4 niveles de Kirkpatrick ${ }^{35}$. El nuevo proyecto se inserta en este contexto, incluyendo el desarrollo y validación del instrumento CAJEP y con la información obtenida del análisis de necesidades, la dirección de postgrado desarrollará un plan de capacitación docente focalizado, orientado a ofrecer herramientas útiles y relevantes para el desempeño de los JPEM. De esta manera, la dirección de postgrado y los JPEM de la FM-PUC, podrán realizar reformas curriculares con el objeto de mejorar de manera focalizada, aspectos deficientes que hayan sido identificados mediante las encuestas de medición del ambiente educacional, para tomar acciones de manera racional.

En conclusión, consideramos que el uso de metodología cualitativa y cuantitativa, entrega información complementaria que enriquece el análisis de necesidades de cada institución que desee implementar un programa de capacitación docente. Los JPEM participaron activamente, mostrando su interés en una posterior capacitación. El CAJEP es un instrumento válido y confiable, de gran utilidad en el desarrollo de programas de postgrado de diferentes especialidades y subespecialidades médicas y recomendamos su uso en instituciones de habla hispana para el reconocimiento de las necesidades locales.

\section{Referencias}

1. Robin BR, PhD, McNeil SG, EdD, Cook DA, MD, MHPE, Agarwal KL, MD, Singhal GR, MD, MEd Preparing for the Changing Role of Instructional Technologies in Medical Education. Acad Med 2011; 86 (4): 435-9.

2. Davis DA, MD, Prescott J, MD, Fordis CM, Jr., MD, Greenberg SB, MD, Dewey CM, MD, Brigham T, MDiv, $\mathrm{PhD}$, et al. Rethinking CME: An Imperative for Academic Medicine and Faculty Development. Acad Med 2011; 86 (4): 469-73.

3. Centra JA. Types of faculty developmnent programs. Journal of Higher Education 1978; 49 (2): 151-62.

4. Bland C, Schmitz C, Stritter F, Henry R, Aluise J. Successful faculty in academic medicine. New York: SpringerVerlag; 1990.

5. Sheets KJ, Schwenk TL. Faculty development: a field of dreams. Acad Med 1990; 2: 141-8.

6. Holmboe E, Ward D, Reznick R, Katsufrakis P, Leslie K, Patel V, et al. Faculty Development in Assessment: The Missing Link in Competency-Based Medical Education. Acad Med 2011; 86 (4): 460-7.

7. Amin Z, Eng KH, Gwee M, Hoon TC, Rhoon KD. Addressing the needs and priorities of medical teachers through a collaborative intensive faculty development programme. Med Teach 2006; 28: 85-8.

8. ACGME. Accreditation Council for Graduate Medical Education [Internet] 1999-2007 [Consulta el 11 de Mayo de 2011]. Disponible en: http://www.acgme.org/ acWebsite/home/home.asp.

9. Cumming A, Ross M. The Tuning Project for Medicinelearning outcomes for undergraduate medical education in Europe. Med Teach 2007; 29: 636-41.

10. Riquelme A, Méndez B, de la Fuente P, Padilla O, Benaglio $C$, Sirhan $M$, et al. Desarrollo y validación de encuesta de percepción de portafolio en estudiantes de medicina de pregrado. Rev Med Chile 2011; 139 (1): 4553.

11. Andresen M, Riquelme A, Hasbún P, Díaz C, Montaña R, Regueira T. Evaluación de competencias de intubación traqueal mediante un escenario simulado en internos de medicina. Rev Med Chile 2011; 139 (2): 165-70.

12. Bitran M, Mena B, Riquelme A, Padilla O, Sánchez I, Moreno R. [An instrument in Spanish to evaluate the performance of clinical teachers by students]. Rev Med Chile 2010; 138 (6): 685-93.

13. Soemantri D, Herrera C, Riquelme A. Measuring the educational environment in health professions studies: a systematic review. Med Teach 2010; 32 (12): 947-52.

14. Triviño X, Vásquez A, Mena A, López A, Aldunate M, Varas M. [Objective Structured Clinical Examination 
for a pediatric internship assessment in two schools of medicine]. Rev Med Chile 2002; 130: 817-24.

15. Delfino AE, Chandratilake M, Altermatt FR, Echevarría G. Validation and piloting of direct observation of practical skills tool to assess intubation in the Chilean context. Med Teach 2013 Feb 27. [Epub ahead of print].

16. Varas J, Mejía R, Riquelme A, Maluenda F, Buckel E, Salinas J, et al. Significant transfer of surgical skills obtained with an advanced laparoscopic training program to a laparoscopic jejunojejunostomy in a live porcine model: feasibility of learning advanced laparoscopy in a general surgery residency. Surg Endosc 2012 Dec; 26 (12): 3486-94.

17. Ullian AJ, Stritter FT. Types of faculty development programs. Family Medicine 1997; 29 (4): 237-41.

18. O'Cathain A, Murphy E, Nicholl J. Three techniques for integrating data in mixed methods studies. BMJ 2010; 341: c4587.

19. Hesketh EA, Bagnall G, Buckley EG, et al. A framework for developing excellence as a clinical educator. Medical Education 2001; 35: 555-64.

20. Adkoli BV, Al-Umram KU, Al-Sheikh MH, Deepak KK. Innovative Method of Needs Assessment for Faculty Development Programs in a Gulf Medical School. Education for Health (Abingdon) 2010; 23 (3): 389: e1-12.

21. Cronbach LJ. Coefficient alpha and the internal structure of tests. Psychometrika 1951; 16 (3): 297-334.

22. Strauss A, Corbin J. Basics of Qualitative Research: Grounded Theory Procedures and Techniques. Thousand Oaks, CA: Sage; 1990.

23. Ruiz J. Metodología de la investigación cualitativa Bilbao: Universidad de Deusto; 1995.

24. Steinert Y. Commentary: Faculty Development: The Road Less Traveled. Academic Medicine 2011; 86 (4): 409-11

25. Steinert Y, Nasmith L, McLeod P, Conochie L. A teaching scholars' program to develop leaders in medical educa- tion. Acad Med 2003; 78: 142-9.

26. Baxley EG, Probst JC, Shell BJ, Bogdewic SP, Cleghorn GD. Program-centred education: a new model for faculty development. Teaching and Learning in Medicine 1999; 11: 94-9.

27. Flynn SP, Bedinghaus J, Snyder C, Hekelman F. Peer coaching in clinical teaching: a case report. Educational Research and Methods 1994; 26: 569-70.

28. Montero L, Triviño X, Sirhan M, Moore P, Leiva L. [Barriers for faculty development in medical education: a qualitative study]. Rev Med Chile 2012; 140 (6): 695702.

29. Rubeck RF, Witzke DB. Faculty Development: a field of dreams. Acad Med 1998; 73: 33-7.

30. Searle NS, Thibault GE, Greenberg SB. Faculty Development for Medical Educators: Current Barriers and Future Directions. Acad Med 2011; 86 (4): 405-6.

31. Herrera C, Olivos T, Román JA, Larraín A, Pizarro M, Solís N, et al. Evaluación del ambiente educacional en programas de especialización médica. Rev Med Chile 2012; 140: 1554-61.

32. Riquelme A, Padilla O, Herrera C, Olivos T, Román JA, Sarfatis A, et al. Development of ACLEEM questionnaire, an instrument measuring residents' educational environment in postgraduate ambulatory setting. Med Teach 2013; 35 (1): e861-6.

33. O'Sullivan P, Irby D, Reframing Research on Faculty Development. Acad Med 2011; 86 (4): 421-8.

34. Steinert Y. Faculty development in the new millennium: key challenges and future directions. Med Teach 2000; 22: 44-50.

35. Kirkpatrick DL. Evaluating teaching program. San Francisco: Berret-Koehler Publishers; 1994.

36. Sirhan M, Triviño X. [Evaluation of a faculty development program in curriculum development for program directors of medical specialties]. Rev Med Chile 2012; 140 (4): 530-7. 